\title{
Bit Error Rate Analysis of Mobile Ad Hoc Networks over $\eta-\mu$ Fading Channels
}

\author{
Ibrahim Ghareeb, Amani Atiani*
}

Department of Electrical Engineering, Jordan University of Science \& Technology, Irbid, Jordan.

*Email: amaniatiani@gmail.com

Received 13 July 2014, Accepted 7 September 2014, Published 14september 2014

\begin{abstract}
In this paper the performance analysis of Mobile ad hoc networks (MANETs) is conducted for a differential QPSK (DQPSK) signals with post-detection equal gain combining (EGC) receiver operating over additive white Gaussian noise (AWGN) as well as for slow frequency nonselective $\eta-\mu$ fading channels, in which the diversity branches can have unequal signal-to-noise ratios (SNRs) as well as different severity parameters. The average bit error probability (ABEP) is evaluated using MGF-based approach. The average BER per multi-hop route of MANETs for this communication is studied.
\end{abstract}

Keywords: $\eta-\mu$ fading channels, EGC, DQPSK signals, MANETs, INI, DP mobility model, ONRBS, RBS.

\section{Introduction}

Ad hoc networks consist of sets of transmitting and receiving nodes distributed randomly in space, having the responsibility to transmit, relay, and receive data packets without a centralized control. These networks have the advantage of avoiding the cost, installation and maintenance of network infrastructure, being available to be rapidly deployed and reconfigured, and exhibiting great robustness owing to their distributed nature, node redundancy and lack of single points of failure. Naturally, these desirable properties introduce many challenges in the design of such wireless networks, thus placing this field of study under extensive research for several decades. Recently, the nodes are very likely to be mobile which means that a new challenge appears. In MANETs, the network topology becomes time varying so the network design should guarantee that the route will continue to work after the link is broken. However, the analytical framework which is introduced for static nodes can be extended to cope with the node mobility. The evaluation of the system performance will be carried out for (Ferrari et al., 2004):

1- An ideal network-communication scenario without INI.

2- A realistic network-communication scenario where the communication is affected by in- ter-node-interference (INI). In the presence of INI, two MAC protocols are considered:

a- Reserve-and-go (RESGO) MAC protocol, where the node after reserving a route to its destination, transmits without sensing the channel. In Ferrari and Tonguz (2003a), this protocol is called Aloha MAC protocol since it resembles the classical Aloha in terms of the independent activation of the route from the other nodes activity. However, there are significant differences make this MAC protocol different from the classical Aloha MAC protocol, which are multi-hop route reservation and no use of retransmission techniques.

b- Reserve-listen-and-go (RESLIGO) MAC protocol, where the node after reserving a route to its destination, senses the channels before transmitting, once the channel is idle, the node can start transmitting, if the source node finds that another route is active, it delays the activation of its reserved route. In Ferrari and Tonguz $\left(2003^{a}\right)$, this MAC protocol is called per-route carrier sense multiple access (PRCSMA) MAC protocol, for its resemblance with classical CSMA MAC protocol. In ad hoc wireless network with a number of nodes not extremely large and a high receiver sensitivity, we assume that every node can hear the other nodes in the network, using this MAC protocol results that at any time, there is only one active route in the network, which means that the interference between nodes is re- 
duced to zero, and the network performance is similar to that in the ideal case. In this study, the analysis is confined to the ideal and realistic-RESGO communication scenarios. The results corresponding to the ideal case are valid when RESLIGO MAC protocol is considered (Ferrari and Tonguz, 2007), since the interference between nodes is reduced basically to zero.

\section{Network model and assumptions}

In this study, we will adopt a communication scenario that is a hybrid of circuit switching and packet switching. In this scenario, a multi-hop communication route between the source node and the destination node is initially created through a route discovery mechanism based on broadcast percolation (Ferrari and Tonguz, 2003 ${ }^{\mathrm{b}}$ ). The nodes through the created route are reserved for this transmission only, as in the circuit switching technique. Instead of transmitting data continuously as in pure circuit switching, the data is packetized with an average number of packets transmitted per unit time $\lambda$ (pck/s). This will reduce the interference and allowing the random access scheme without retransmission. The packet transmission is assumed to be Poisson distributed with parameter $\lambda$, then the average inter-arrival rate between two consecutive packets is $1 / \lambda$, the packet duration $L / R b$ (where $L$ (b/pck) is the packet length and $R b(\mathrm{~b} / \mathrm{s})$ is the transmission data-rate) should be sufficiently smaller than $1 / \lambda$, to get disjoint and non-overlap routes which means that the inter-route interference will be significantly reduced (Ferrari and Tonguz, 2003a).

The characteristics of the adopted communication scenario can be summarized as follows (Ferrari et al., 2004)

- The route creation phase will not be explicitly considered. We will assume that it is perfectly accomplished.

- Peer-to-Peer multi-hop communication with nonoverlap routes is considered, which means that no node can act as relay node in more than one route at the same time.

- Each node has a fixed transmitted power $P$, and the routes are constituted with minimum length links.
- The nodes have no buffers; if a node needs to communicate with other nodes, it must first reserve a multi-hop route then it can transmit.

- At each node, the packet transmission/generation can be described by a Poisson process with parameter $\lambda(\mathrm{pck} / \mathrm{s})$. Each generated packet has a fix length of $L$ bits. The condition $\lambda L \leq R b$ has to be satisfied in order that, the proposed communication scenario works properly.

- Retransmission mechanism, such as automatic-repeat-request (ARQ) is not allowed. This assumption is valid for real-time applications, where the delay and the node battery power are very important.

- It is assumed that there are $\mathrm{N}$ mobile nodes in the network of area $A$. The nodes are likely to move inside the area $A$ and never leave, such as mobile phones and laptops in an airport terminal or university campus (Ferrari and Tonguz, 2007).

- All nodes always have packets available for transmission. Also, each relay node in a multi-hop route will have a lower probability of transmitting with respect to the source node, because of the presence of idle interval during which no packet is received from the preceding node.

\section{Network topology}

In order to describe Ad hoc networks, no specific assumption should be made regarding to the network topology, where the nodes have to be randomly placed in the network. However, we will strict our analysis to an ad hoc network with regular square grid topology, to make the derivation tractable. We will also assume the nodes are static, and then the mobile nodes will be considered in the next section. We assume that $N$ nodes are placed uniformly at the vertices of a square grid over a circular surface with area $A$; each node has four nearest neighbors as shown in Figure (1). From Figure (1), $A \approx(\sqrt{ } N$ dlink )$^{2}$, where dlink is the link length which is the distance between any two neighboring nodes. Let's define the node spectral density of the network as $\rho s=N / A$, then dlink can be written as

$$
d_{\text {link }}=\frac{1}{\sqrt{\rho_{s}}}
$$




\section{Communication-theoretic preliminaries}

\section{Average Number of Hops}

Since we consider multi-hop ad hoc networks, the average number of hops per route is an important parameter in order to evaluate the network performance. Consider a multi-hop route with number of hops $n_{h}$, such that $n_{h} \leq n_{h}^{\max }$, where $n_{h}^{\max }$ is the maximum number of hops. Obviously, $n_{h}$ is an integer RV lies between (Ferrari et al., 2007, $n_{h}^{\max }$ ), by assuming that the source node and the destination node lie at opposite ends of a diameter over circular surface, with large number of nodes in the network area $A$ which implies that the deviation from the straight line is limited. Consequently, $n_{h}^{\max }$ can be expressed as Ferrari and Tonguz (2003 a)

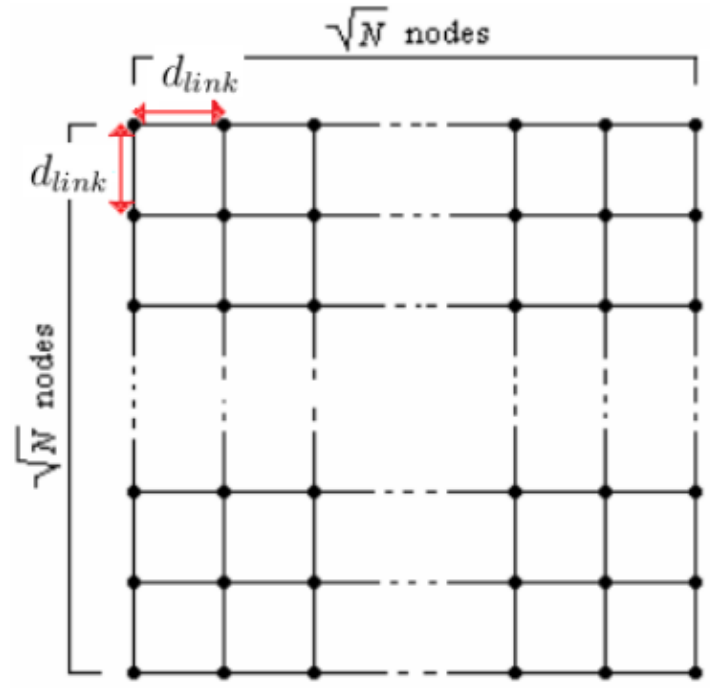

Figure 1. Square grid topology

$$
\begin{aligned}
n_{h}^{\max } & =\left\lfloor\frac{\text { diameter of a circle with area } A}{d_{\text {link }}}\right\rfloor \\
& =\left\lfloor 2 \sqrt{\frac{N}{\pi}}\right\rfloor
\end{aligned}
$$

By assuming that $n_{h}$ has any symmetric distribution, as uniform distribution which is given by $f_{n_{h}}(x)=1 / n_{h}^{\max }$, for $0<x<n_{h}^{\max }$, and zero otherwise. Hence $\frac{\dot{n}}{n_{h}}$ is given by

$$
\overline{n_{h}}=\frac{n_{h}^{\max }}{2}=\left\lfloor\sqrt{\frac{N}{\pi}}\right\rfloor
$$

\section{Average Bit Error Rate per Route}

By assuming that there is regeneration at each intermediate node (i.e., detection and possible error correction), also the resultant errors are not corrected in the successive links and accumulate, the BER at the end of the nth link can be expressed as

$$
B E R^{(n)}=1-\left(1-B E R_{\text {link }}\right)^{n}
$$

where $B E R_{\text {link }}$ denotes the $\mathrm{BER}$ at the end of a single link, which is related to the link SNR $\left(S N R_{\text {link }}\right)$ at the receiving node by a function depends on the modulation format, channel coding scheme, and the fading channel model. In this paper, we will consider $\eta-\mu$ fading channel with uncoded transmission (Atiani, 2013). Hence, the average BER per route can be expressed as (Ferrari et al., 2003).

$$
\overline{B E R}=B E R^{\left(\overline{n_{h}}\right)}=1-\left(1-\overline{B E R}_{\text {link }}\right)^{\overline{n_{h}}}
$$

where $\overline{B E R}_{\text {link }}$ is the average BER at the end of a single link.

\section{Signal-to-noise Ratio per Link}

Assuming that the transmitted signal is affected by free-space loss, then referring to Friis formula (Rappaport, 2002), the received power at the end of a single link $P r$, can be expressed as (Ferrari and Tonguz, 2003 ${ }^{\mathrm{b}}$ )

$$
P_{r}=\frac{\nu P_{t}}{d_{\text {link }}^{2}}=\frac{G_{t} G_{r} \lambda_{c}^{2}}{(4 \pi)^{2} f_{l} d_{\text {link }}^{2}} P_{t}
$$

where $P t$ is the transmitted power which is assumed to be common for all nodes. Gt and Gr are the transmitter and receiver antenna gains, respectively. $\lambda_{c}=c / f_{c}$ is the wavelength corresponding to the carrier frequency $f_{c}, c$ is the speed of light and $f_{l} \geq 1$ is the loss factor. Although, we consider the scenario with free space propagation only, where $P_{r} \propto P_{t} / d_{\text {link }}^{2}$ , the analysis can be extended to the other propagation scenarios characterized by stronger attenuation, where $P_{r} \propto P_{t} / d_{\text {link }}^{x}, x>2$ (Rappaport, 2002). We will consider two cases, based on the absence or presence of the interference between nodes (Ferrari and Tonguz, 2007). 


\section{Ideal Case}

In an ideal scenario, there is no INI, and the only affecting noise is the thermal noise, which is assumed as an additive white Gaussian noise. Hence, the average link SNR (averaged with respect to fading) is given by

$$
\overline{S N R}_{\text {link }}=\mathcal{E}\left[\alpha^{2}\right] \frac{E_{b}}{N_{0}}=\mathcal{E}\left[\alpha^{2}\right] \frac{\nu P_{t}}{F k T_{0} R_{b} d_{\text {link }}^{2}}
$$

where $\mathcal{E}[$.$] denotes the expected value,$ $E_{b}=P_{r} / R_{b}$ is the received energy per bit, and $N_{0}=F k \dot{T}_{0}$ is the thermal noise power spectral density, where $\mathrm{F}$ is the noise figure, and $k=1.38 \times$ $10^{-23} \mathrm{~J} / \mathrm{K}$ is the Boltzmann's constant, and $T 0=300$ $\mathrm{K}$ is the room temperature, $\alpha$ is the fading channel envelope and $\nu=\frac{G_{t} G_{r} c^{2}}{(4 \pi)^{2} f_{L} f_{c}^{2}}$. This case can be practically holds in a network where different multi-hop routes does not interfere with each other, which can be obtained, for example, by using perfectly orthogonal spreading codes or disjoint frequency bands in active disjoint multi-hop routes.

\section{Realistic Case}

In this case the nodes interfere with each other, we will assume that the interfering signals can be treated as additive white noise which is independent from the thermal noise, and then the link SNR in the presence of INI can be written as (Ferrari and Tonguz, 2003a; Ferrari et al., 2004).

$$
\overline{S N R}_{\text {link }}=\mathcal{E}\left[\alpha^{2}\right] \frac{\nu P_{t} / d_{\text {link }}^{2}}{F k T_{0} R_{b}+\zeta P_{I N T}}
$$

where $\zeta$ is the spectral efficiency which is defined as $\zeta=R_{b} / B_{T}$. Assuming that $\zeta=\log _{2} M$, where $M$ is the cardinality of the transmitted signal; this assumption is valid for the considered modulation format (Ferrari et al., 2004). It is obvious from (8) that $\zeta$ acts as amplification factor; higher $\zeta$ means larger impact of the INI.

\section{Interference Power}

The interference power PINT depends on the network topology and the specific MAC protocol. Since in the RESLIGO MAC protocol scenario, the interference power is approximately zero, and our analysis is limited to the RESGO MAC protocol scenario. In order to provide an expression for PINT for a reg- ular square grid topology, we assume that the source node $n d$ is placed at the center of the network, and the nodes around it can be grouped in tiers of increasing order as shown in Figure (2) (Ferrari and Tonguz, 2007). Since the number of nodes in the network is finite, there exists a maximum tier order imax which depends on the number of nodes $N$. There are $8 \mathrm{i}$ nodes on the ith order tier, then

$$
N \approx \sum_{i=1}^{i_{\max }} 8 i=4 i_{\max }\left(i_{\max }+1\right)
$$

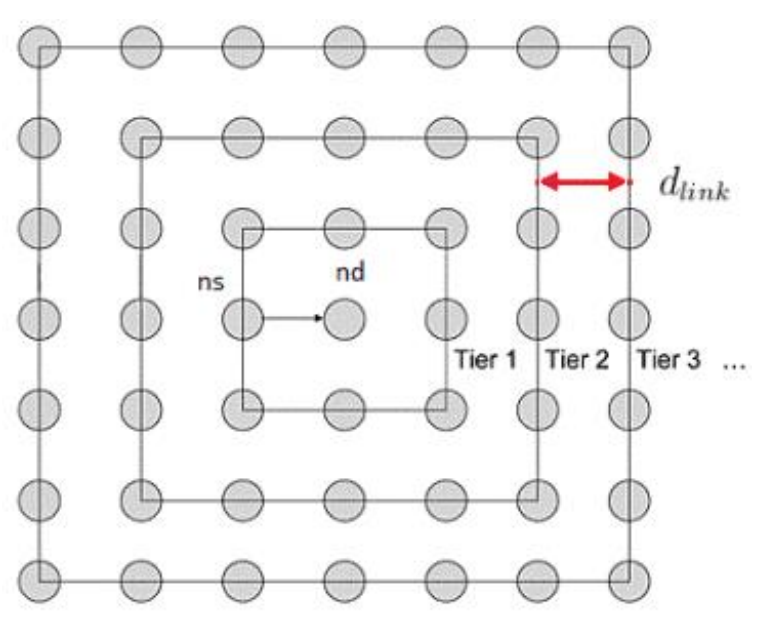

Figure 2. Tiered structure of nodes in case of regular square-grid topology.

Due to the particular shape of the network surface, not all tiers are fully filled with nodes. Also, by assuming that $N$ is sufficiently large, equation (9) becomes

$$
N \approx 4 i_{\max }^{2} \Rightarrow i_{\max } \approx\left\lfloor\frac{\sqrt{N}}{2}\right\rfloor
$$

To determine PINT, we need to know the probability that the interfering node is effectively transmitting, which is depends on the MAC protocol. This probability is used to weight the received power of the interfering node. However, the packet transmission at each node is a Poisson distributed with average packet transmission rate $\lambda$, such as

$$
\begin{array}{r}
P(\text { a node transmits k packets in t seconds) } \\
=\frac{(\lambda t)^{k}}{k !} \exp (-\lambda t)
\end{array}
$$

The main features of RESGO MAC protocol, is that the source after reserving the multi-hop route to its destination, immediately starts to transmit without 
sensing the channel. Also, this proposed MAC protocol does not use retransmission mechanisms to avoid collisions, so a bit level interference analysis is more accurate to guarantee the required QoS. We need to define the vulnerable interval of a generic node, which is the interval that any message transmission from this node, starts within this interval will interfere with the considered bit in the message that is transmitted by the source. In the case with RESGO MAC protocol, the length of the vulnerable interval is $D p c k=L / R b$, and does not depend on the specific bit position in the transmitted message. Thus, we will focus on the probability of a single bit in the transmitted signal, interferes with the transmission from the other nodes, which does not depends on either the particular bit in the transmitted message or on the particular interfering node, so Ferrari and Tonguz (2007)

$$
p=1-\exp \left(-\lambda D_{p c k}\right)
$$

Hence, PINT can be expressed as Tonguz and Ferrari (2006)

$$
P_{I N T} \approx \nu \frac{P_{t}}{d_{\text {link }}^{2}}\left(1-\exp \left(-\lambda D_{p c k}\right)\right) \triangle_{A}(N)
$$

where

$$
\triangle_{A}(N)=\sum_{i=1}^{\lfloor\sqrt{N} / 2\rfloor}\left(\frac{6}{i^{2}}+8 \sum_{j=1}^{i-1} \frac{1}{i^{2}+j^{2}}\right)-1
$$

The quantity $\triangle_{A}(N)$ depends on the network geometry. In Tonguz and Ferrari (2006), it is shown that the difference between various uniform topologies is negligible, since there is an excellent agreement between the exact BER per route and the approximated one for the considered network scenarios. However, for low traffic load $\lambda D p c k<1$, then $1-\exp (-\lambda D p c k) \approx \lambda D p c k$. Hence, equation (13) can be approximated as

$$
P_{I N T} \approx \nu \frac{P_{t}}{d_{\text {link }}^{2}} \lambda D_{p c k} \triangle_{A}(N)
$$

\section{Direction-persistent (DP) mobility models}

Ad hoc networks have the ability to cope with mobility; the analytical framework introduced above for static nodes can be extended to include the mobile nodes, under constraint that the nodes movement is confined within the fixed network surface. The mobility status of a node is described by two random processes: speed $v$ and direction angle $\theta$ (with respect to a horizontal axis). Many switching/routing strategies can be considered as Ferrari and Tonguz (2006):

1- Opportunistic non-reservation-based switching (ONRBS), where the source node does not statically reserve intermediate relay nodes. Instead, the consecutive links are chosen opportunistically based on their lengths (i.e., consecutive links of length dlink $=1 / N \rho$ s are chosen). In other words, if two successive nodes move too far from each other, the starting node of this link will choose another node at average distance dlink. It is always assumed that all nodes are confined within the network surface of fixed area, and a node can always finds a neighboring node at distance dlink. Depending on the definition above, the network that using ONRBS strategy is not affected by the nodes mobility.

2- Reservation-based switching (RBS), where successive hops of a multi-hop route are activated after they are discovered, and reserved regardless of their actual length due to mobility, even it is assumed that the initial setup of this multi-hop route is characterized by a sequence of hops of length dlink. Also, it is assumed that the mobility status of each node remains constant until the entire packet being transmitted, where an intermediate node cannot start forwarding the packet, before having received it completely. However, it is expected that ONRBS is more robust than RBS against mobility model. Various node mobility models have been proposed to describe node mobility in MANETs (Ghareeb and Abu Al-Hajja, 2008), which can be basically involved in the following two models Ferrari and Tonguz (2007):

1- Direction-persistent (DP) mobility model; where the direction and speed of the movement of the two nodes at the end of the link are constant.

2- Direction-non-persistent (DNP) mobility model, in this scenario the mobile node can change the movement during the packet transmission. Here, evaluating the performance of MANETs will be confined on the DP mobility model; our analysis can be extended to consider the other model in a straightforward manner (Ferrari and Tonguz, 2007). However, the DP mobility model is also called the Random Walk mobility model (Zonoozi and Dassanayake, 1997). In this model, the speed of each mobile node is uniformly distributed between $[0$, $\checkmark \max ]$, also the direction is chosen uniformly from the interval $[0,2 \pi]$. The change in the distance between 
the source node and the destination node is calculated and used to evaluate the network performance.

Under assumption of DP mobility model, we consider an intermediate link in a multi-hop route, where the two nodes are $n_{A}$ and $n B$, referring to the adopted mobility model, the two node have constant speeds and direction angles, which are $\left(V_{A}, \theta_{A}\right)$ and $\left(V_{B}, \theta_{B}\right)$, respectively, during the packet transmission. The initial link length is $d_{\text {link }}^{s}=d_{\text {link }}$ and the final link length is $d_{\text {link }}^{e}$, and the packet transmission starts at $t$ $=t s$ and ends at $t=t e=t s+D p c k$ as shown in Figure (3).

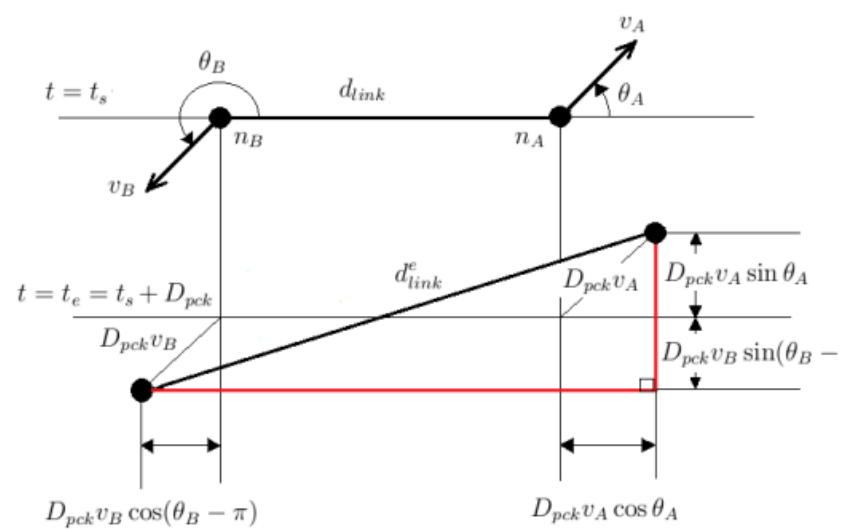

Figure 3. Link evolution during a packet transmission for DP mobility model.

By using simple geometric considerations, $d_{\text {link }}^{e}$ can be expressed as Ferrari and Tonguz (2007)

$$
\begin{aligned}
\left(d_{\text {link }}^{e}\right)^{2} & =d_{\text {link }}^{2}+D_{p c k}^{2}\left(v_{A}^{2}+v_{B}^{2}\right) \\
& -2 v_{A} v_{B} D_{p c k}^{2} \cos \left(\theta_{A}-\theta_{B}\right) \\
& +2 d_{\text {link }} D_{p c k}\left(v_{A} \cos \theta_{A}-v_{B} \cos \theta_{B}\right)
\end{aligned}
$$

In order to evaluate the average network performance. we need to compute the average link length $\overline{\bar{d}}_{\text {link }}$, which is given by

$$
\begin{aligned}
\bar{d}_{\text {link }}= & \frac{d_{\text {link }}^{s}+d_{\text {link }}^{e}}{2} \\
= & \frac{d_{\text {link }}}{2} \\
+ & \frac{1}{2}\left[d_{\text {link }}^{2}+D_{p c k}^{2}\left(v_{A}^{2}+v_{B}^{2}\right)\right. \\
& -2 v_{A} v_{B} D_{p c k}^{2} \cos \left(\theta_{A}-\theta_{B}\right) \\
& \left.+2 d_{\text {link }} D_{p c k}\left(v_{A} \cos \theta_{A}-v_{B} \cos \theta_{B}\right)\right]^{1 / 2}
\end{aligned}
$$

However, all equations that are obtained previously in static nndes scenario can be valid for mobile nodes, if $\bar{d}_{\text {link }}$ is replaced by $\bar{d}_{\text {link }}$. The major difference between the two scenarios is that for the mobile nodes, $\overline{\bar{l}}_{\text {link }}$ is a RV whose realization depends on the realization of $\left(V_{A}, \theta_{A}\right)$ and $\left(V_{B}, \theta_{B}\right)$.

\section{DP Mobility Model with ONRBS}

In this strategy, it is assumed that the successive links in the multi-hop route are independent of each other. In other words, the mobility status of a node during a packet transmission is independent of its mobility status during the packet transmission in the consecutive link.

\section{DP Mobility Model with RBS}

In Ferrari and Tonguz (2007), the reserved multi-hop route is described as a tube that bends due to the node mobility. However, this tube may be going to break and needs to be updated by the routing protocol. It is obvious that the higher the speed, the sooner that the tube is going to break. Equations (16) and (17) can be easily extended to the RBS case. For each link of the multi-hop route, we compute the corresponding starting and final lengths, to obtain the average link length. In general, for the ith link between $n i$ and $n_{i+1}$, which is activated at time instant $t=t 1+(i-1) D p c k$ for $i=1,2, \ldots, \bar{n}_{h}$, thus the starting, the final, and the arithmetic mean lengths are given respectively, as 


$$
\begin{aligned}
d_{\text {link }}^{(i, s)}= & {\left[d_{\text {link }}^{2}+\left((i-1) D_{p c k}\right)^{2}\left(v_{i}^{2}+v_{i+1}^{2}\right)\right.} \\
& -2 v_{i} v_{i+1}\left((i-1) D_{p c k}\right)^{2} \cos \left(\theta_{i}-\theta_{i+1}\right) \\
& +2 d_{\text {link }}(i-1) \\
& \left.\times D_{p c k}\left(v_{i} \cos \theta_{i}-v_{i+1} \cos \theta_{i+1}\right)\right]^{1 / 2}(18) \\
d_{\text {link }}^{(i, e)}=[ & d_{\text {link }}^{2}+\left(i D_{p c k}\right)^{2}\left(v_{i}^{2}+v_{i+1}^{2}\right) \\
& -2 v_{i} v_{i+1}\left(i D_{p c k}\right)^{2} \cos \left(\theta_{i}-\theta_{i+1}\right) \\
& \left.+2 d_{\text {link }} i D_{p c k}\left(v_{i} \cos \theta_{i}-v_{i+1} \cos \theta_{i+1}\right)\right]^{1 / 2}
\end{aligned}
$$

$$
\bar{d}_{\text {link }}^{(i)}=\frac{d_{\text {link }}^{(i, s)}+d_{\text {link }}^{(i, e)}}{2}
$$

However, it is assumed that the propagation time between two neighboring nodes is negligible compared to the duration of a packet transmission. This assumption is valid in almost any practical ad hoc wireless networks (Ferrari and Tonguz, 2007).

\section{Numerical results}

Through this section, we will assume the following [5], Ghareeb and Abu Al-Hajja (2008):

- $c=3 \times 10^{8} \mathrm{~m} / \mathrm{s}$ is the speed of light

- $k=1.38 \times 10^{-23}$ is the Boltzman's constant

- $T 0=300 \mathrm{~K}$ is the room temperature in Kelvin

- $f_{c}=2.4 \mathrm{GHz}$ is the carrier frequency

- $f l=1$ is the loss factor

- $G t=G r=1$ are the transmitter and receiver antenna gains, respectively (omni-directional antennas)

- $F=6 d B$ is the noise figure

- $R b=2 \mathrm{Mb} / \mathrm{s}$ is the transmission data-rate

- $\quad N=1000$ is the number of nodes.

However, all other parameters will be given on the figures and independent $\eta-\mu$ fading channels are considered (Atiani, 2013). Figure (4) studies the impact of mobility on the BER for DP model with RBS at fixed packet length $\left(10^{6}\right)$ bits, with ideal (no INI) case. It is obvious that the mobility degrades the system performance, since higher speed means that the distance between nodes increases. Hence, the average SNR per link decreases, and the end to end average BER per multi-hop route degrades. Also, at a fixed speed, an increase in $\rho s$ will improve the system performance, since the nodes become closer to each other. Figure (5) shows the impact of nodes velocities on the average BER per multihop route, using RESGO MAC protocol with DP mobility model and RBS at fixed packet length. Also, it is clear that the impact of node mobility becomes insignificant at higher interference. In realistic scenario with interference, the node mobility becomes irrelevant; the higher the interference between nodes, the lower the impact of the routing protocol. Namely, perfecting the routing protocol without eliminating the INI has no significant effect. Depending on Figure (6), we find that ONRBS is more robust against node mobility; each link is opportunistically activated and dynamically reserved, and the intermediate nodes do not move far away from each other. Figure (7) illustrates the effect of the traffic load and the packet length on the average BER per multi-hop route for MANETs, we find that increasing the packet length degrades the system performance, which is expected. Increasing the packet length will increase the packet duration, which means that the interference power becomes higher. Also, as the packet duration increases, the distance that a node can move increases, then the average BER per multi-hop route degrades. Besides, when the traffic load (average packet transmission rate) increases, the interference power increases, and thus the BER becomes higher. Figure (8) shows that increasing the packet length degrades the BER and the effect is more obvious at higher maximum node velocity $v$ max. For ideal case, increasing the $v \max$ and/or packet length will increase the distance that the node moves during packet transmission, which degrades the average BER per multi-hop route. Figure (9) shows the average BER at the end of a multi-hop route versus $\rho$, for DQPSK signals with post-detection EGC and three independent $\eta$ $\mu$ fading channels at $\mu=1.5$ and $\eta=0.8$, using DP mobility model with RBS. In this Figure, the impact of the packet length and node velocity is studied for realistic scenario with $\lambda=0.001 \mathrm{pck} / \mathrm{s}$. As expected, for shorter packet length, the packet duration is shorter, the interference power is limited, and the distance that a node can move is smaller, therefore, the system performance improves, and the node mobility does not affect the BER significantly. On the other hand, at larger packet size, the interference power becomes significant and degrades the end-to-end BER. Figures (10-12) study the effect of the transmission data-rate on the average BER per multi-hop route for DP mobility model with ONRBS and RBS, using ideal and realistic cases. In Figure (10), we find that there is a threshold value for the node spatial density $\rho$ s, where above this value the network starts to work. Using ONRBS with ideal scenario, decreasing $R b$ will increase SNR, hence the average BER improves. Besides, we find that 
increasing the number of nodes, the network suffers more BER. However, the effect of $R b$ on the average BER suing RBS is shown in Figures (11 and 12). The effect of $R b$ in the case of RBS is not clear as in ONRBS, since ONRBS is very robust against node mobility. An increase in $R b$ reduces the packet duration, and the nodes do not move far away so the BER will be affected less by node mobility. Also, as $R b$ increases the thermal noise will increase and the interference power decreases. Therefore, we need to know which term is dominant. At higher $\rho$ s the interference power becomes dominant; hence the system performance improves when $R b$ increases. However, we can not increase the data-rate arbitrarily in ad-hoc networks, since beyond a critical value the transport capacity decreases significantly (Ferrari and Tonguz, 2007). Figure (13) illustrates the effect of increasing number of nodes on the average BER at the end of a multi-hop route versus $\rho s$, for DQPSK signals with post-detection $E G C$ and three independent $\eta-\mu$ fading channels at $\mu=1.5$ and $\eta=0.8$, in the case of DP mobility model with RBS, at $P t=2 \mathrm{~mW}, v \max =30 \mathrm{~m} / \mathrm{s}$ and packet size $=10000$ bits. As the number of nodes increases, the interference power increases and the end-to-end average BER degrades according to (5). Finally, the most important result we obtain about MANETs, is that designing an efficient MAC protocol that reduced the INI has a greater effect on the system performance than the routing strategy and the mobility level of the nodes. However, reducing the packet length is the best remedy to combat the deleterious effect of the node mobility.

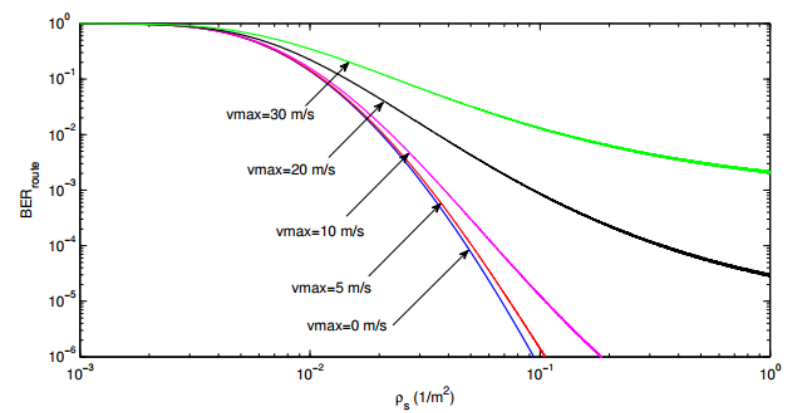

Figure 4. The average BER at the end of a multi-hop route versus $\rho s$, for DQPSK signals with post-detection EGC three independent $\eta-\mu$ fading channels at $\mu=1.5$ and $\eta=0.8$, in the case of DP mobility model with RBS. Ideal (no INI) case is consid-

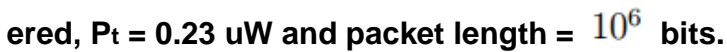

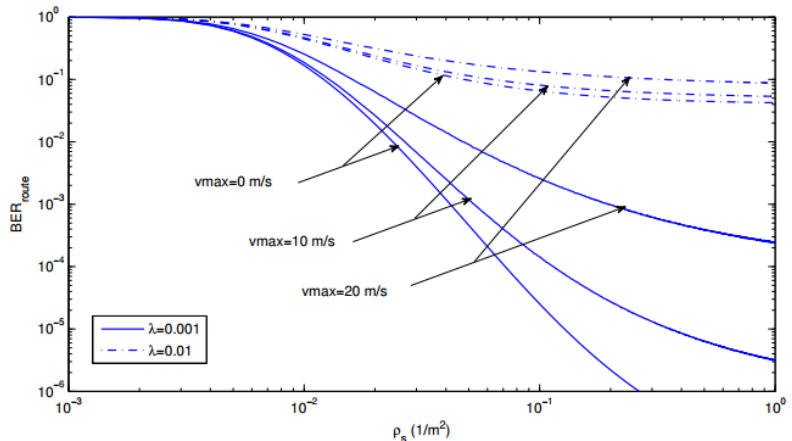

Figure 5. The average BER at the end of a multi-hop route versus $\rho s$, for DQPSK signals withpost-detection EGC and three independent $\eta-\mu$ fading channels at $\mu=1.5$ and $\eta=0.8$, in the case of DP mobility model with RBS. Realistic (with INI) case is

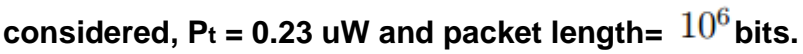

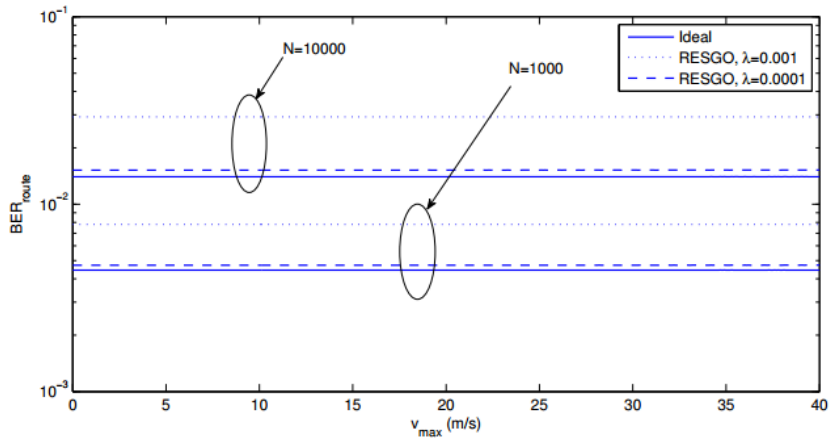

Figure 6. The average BER at the end of a multi-hop route versus Vmax, for DBPSK signals with post-detection EGC and three independent $\eta-\mu$ fading channels at $\mu=1.5$ and $\eta=0.8$, in the case of DP mobility model with ONRBS, $\mathrm{Pt}=2 \mathrm{~mW}$ and $\rho_{s}=2 \times 10^{-6} m^{-2}$

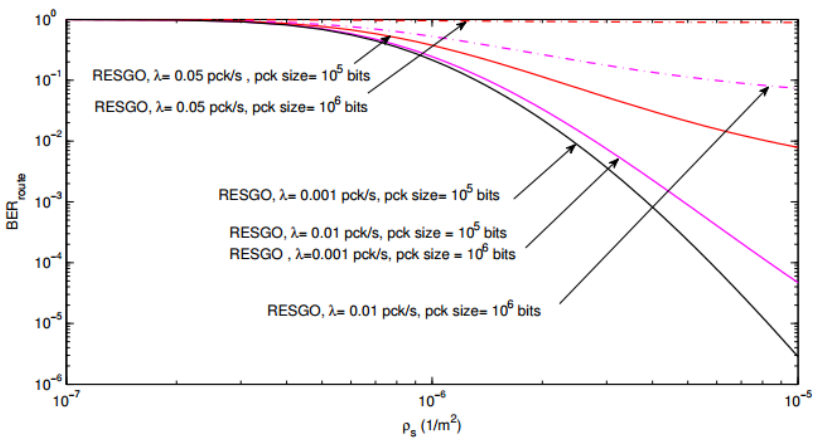

Figure 7. The average BER at the end of a multi-hop route versus $\rho s$, for DQPSK signals with post-detection EGC and three independent $\eta-\mu$ fading channels at $\mu=1.5$ and $\eta=0.8$, in the case of DP mobility model with RBS. $P_{t}=2 \mathrm{~mW}, V_{\max }=30 \mathrm{~m} / \mathrm{s}$. 


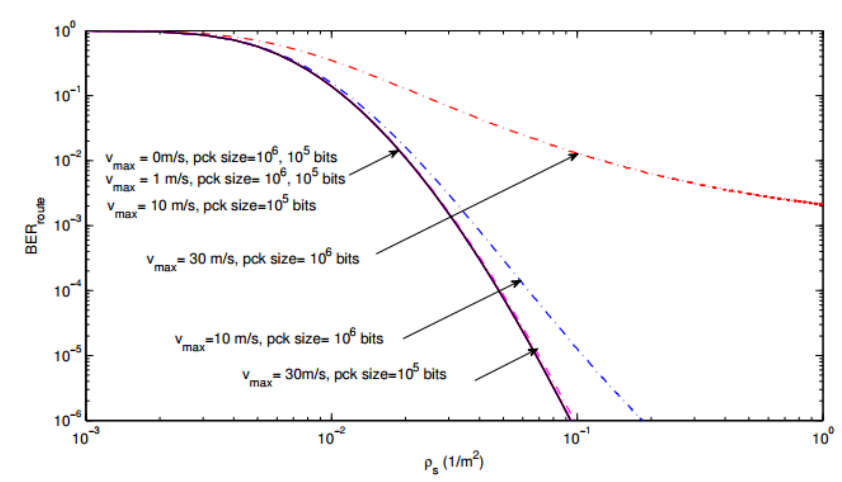

Figure 8. The average BER at the end of a multi-hop route versus $\rho s$, for DQPSK signals with post-detection EGC and three independent $\eta-\mu$ fading channels at $\mu=1.5$ and $\eta=0.8$, in the case of $D P$ mobility model with RBS. Ideal (no INI) scenario is considered and $\mathrm{Pt}_{\mathrm{t}}=0.23 \mathrm{uW}$.

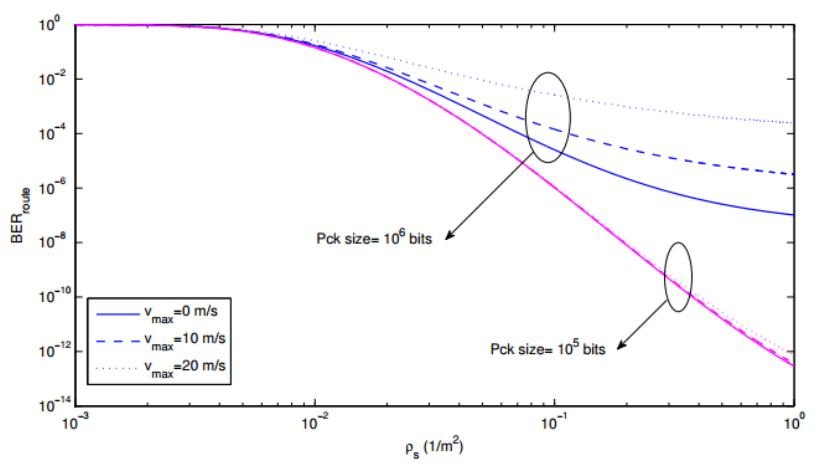

Figure 9. The average BER at the end of a multi-hop route versus $\rho s$, for DQPSK signals with post-detection EGC and three independent $\boldsymbol{\eta}-\boldsymbol{\mu}$ fading channels at $\mu=1.5$ and $\eta=0.8$, using DP mobility model with RBS. Realistic scenario with $\lambda=0.001$ $\mathrm{pck} / \mathrm{s}$ is considered and $\mathrm{Pt}=0.23 \mathrm{uW}$.

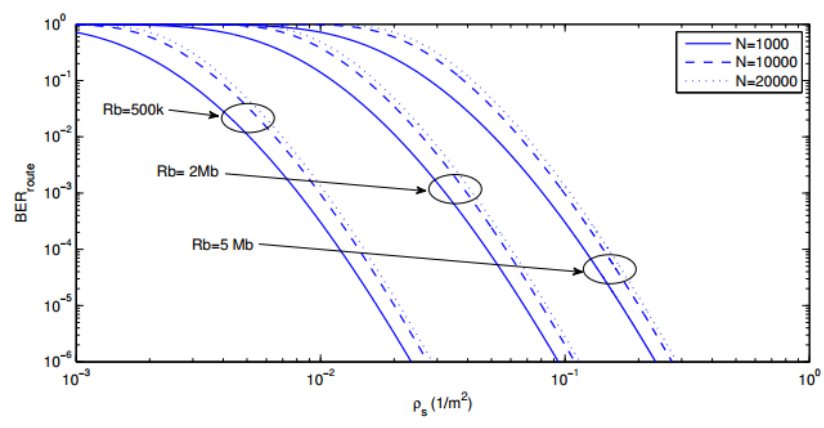

Figure 10. The average BER at the end of a multi-hop route versus $\rho s$, for DQPSK signals with post-detection EGC and three independent $\eta-\mu$ fading channels at $\mu=1.5$ and $\eta=0.8$, in the case of DP mobility model with ONRBS. Ideal (no INI) case is

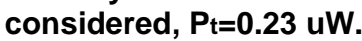

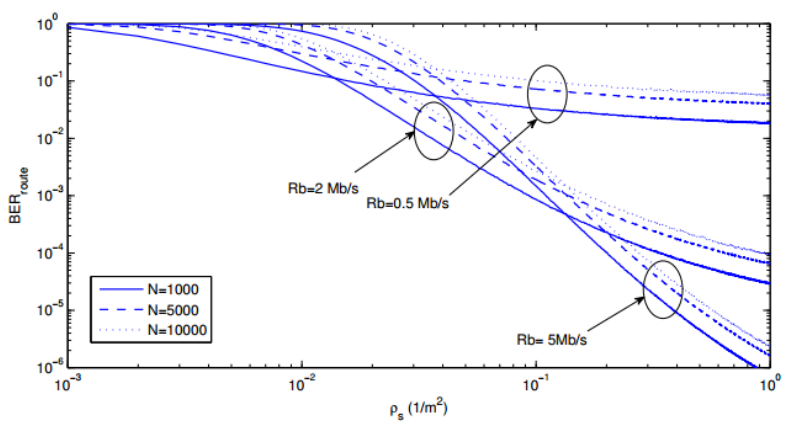

Figure 11. The average BER at the end of a multi-hop route versus $\rho s$, for DQPSK signals with post-detection EGC and three independent $\eta-\mu$ fading channels at $\mu=1.5$ and $\eta=0.8$, in the case of DP mobility model with RBS. Ideal (no INI) case is considered, $\mathrm{Pt}_{\mathrm{t}}=\mathbf{0 . 2 3} \mathrm{uW}$, packet length $=10^{6}$ bits and $\mathrm{v}$ $\max =20 \mathrm{~m} / \mathrm{s}$.

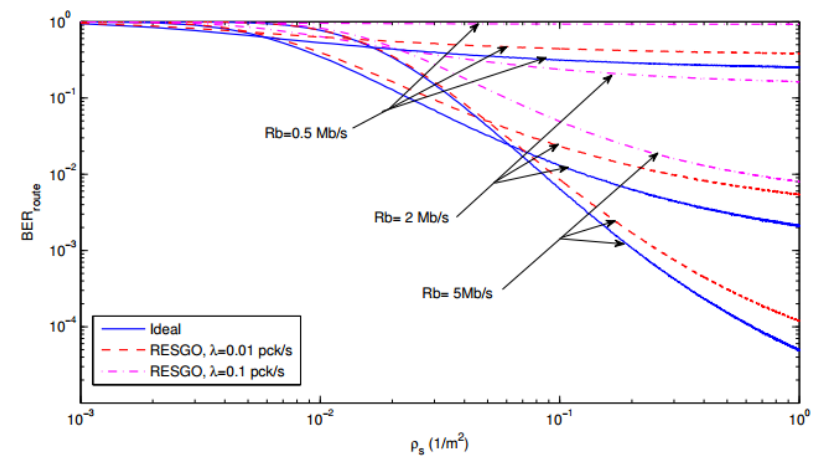

Figure 12. The average BER at the end of a multi-hop route versus $\rho s$, for DQPSK signals with post-detection EGC and three independent $\eta-\mu$ fading channels at $\mu=1.5$ and $\eta=0.8$, in the case of DP mobility model with RBS. $\mathrm{Pt}_{\mathrm{t}}=0.23 \mathrm{uW}$, packet length $=10^{6}$ bits and $V_{\max }=\mathbf{3 0} \mathrm{m} / \mathrm{s}$.

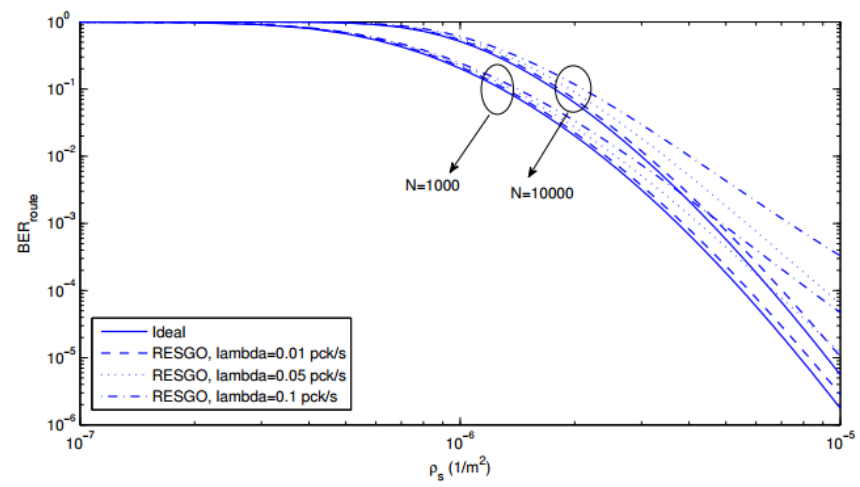

Figure 13. The average BER at the end of a multi-hop route versus $\rho s$, for DQPSK signals with post-detection EGC and three independent $\eta-\mu$ fading channels at $\mu=1.5$ and $\eta=0.8$, in the case of DP mobility model with RBS. $P_{t}=2 \mathrm{~mW}, \mathrm{Vmax}=30 \mathrm{~m} / \mathrm{s}$ and packet size $=10000$ bits. 


\section{Conclusion}

The performance of MANETs is studied by evaluating the average BER per multi-hop route for DQPSK signals with post-detection EGC operating over independent $\eta-\mu$ fading channels. Numerical results have been introduced to explain the effect of mobility, number of nodes, packet length, INI, and traffic load.

The effect of $R b$ in the case of RBS is not clear as in ONRBS, since ONRBS is very robust against node mobility. An increase in $R b$ reduces the packet duration, and the nodes do not move far away so the BER will be affected less by node mobility. Also, as $R b$ increases the thermal noise will increase and the interference power decreases. Therefore, we need to know which term is dominant. At higher $\rho s$ the interference power becomes dominant; hence the system performance improves when $R b$ increases. However, we cannot increase the data-rate arbitrarily in ad-hoc networks, since beyond a critical value the transport capacity decreases significantly.

Finally, the switching/routing plays an important role in ad hoc wireless networks only if the MAC protocol is effective against the interference. If communications in the network are affected by significant interference, then the choice of the switching scheme does not and cannot significantly improve the performance.

\section{References}

Atiani A., Gaussian class multivariate distributions: Theory and applications over mobile fading channels, M.S. thesis, Dept. Elect. Eng., JUST Univ., Irbid, Jordan, 2013.

Ferrari G., Baruffini B. and Tonguz O. K. Spectral efficiency-connectivity tradeoff in ad hoc wireless networks. Proceeding of the International Symposium on Information Theory and its Applications (ISITA'04); 2004 Oct 10-13; Parma, Italy, pp. 451-456.

Ferrari G. And Tonguz O. K. (2007). Impact of mobility on the BER performance of ad hoc wireless networks. IEEE Trans. Veh. Technol. 56(1): 271-286.

Ferrari G. and Tonguz O. K. A communication-theoretic approach to ad hoc wireless networking. Proceedings of Sensor and Ad hoc
Commun. and Networks (SECON); 2006; 2: 715-722.

Ferrari G. and Tonguz O.K. ${ }^{a}$ MAC protocols and transport capacity in ad hoc wireless networks: Aloha versus PR-CSMA. Proceeding of the IEEE Military Communications Conference (IEEE MILCOM); 2003 Oct 13-16; Boston, USA: $1311-1318$.

Ferrari G. and Tonguz O. K. b Performance of ad hoc wireless networks with Aloha and PRCSMA MAC protocols. Proceeding of the IEEE Global Telecommunications Conference Conf. (IEEE GLOBECOM); 2003 Dec; San Francisco, USA: 2824-2829.

Ghareeb I. and Abu Al-Haija A. GMSK modulation over Weibull fading channels and its impact on the effective transport capacity of ad hoc wireless networks. IEEE GLOBCOM Workshops; 2008.

Tonguz O. K and Ferrari G. Ad Hoc Wireless Networks: A Communication-Theoretic Perspective. Wiley; 2006.

Rappaport T. S. Wireless Communications Principles and Practice. 2nd ed. Upper Saddle River, NJ, USA: Prentice-Hall; 2002.

Zonoozi M. M. and Dassanayake P. (1997). User mobility modeling and characterization of mobility patterns. IEEE Journal on Selected Areas in Communications; 15(7): 1239-1252. 